\title{
ANALOGY, SIMILARITY, AND THE PERIODIC TABLE OF ARGUMENTS
}

\begin{abstract}
The aim of this paper is to indicate the systematic place of arguments based on the concept of analogy within the theoretical framework of the Periodic Table of Arguments, a new method for describing and classifying arguments that integrates traditional dialectical accounts of arguments and fallacies and rhetorical accounts of the means of persuasion (logos, ethos, pathos) into a comprehensive framework.

The paper begins with an inventory of existing approaches to arguments based on analogy, similarity and adjacent concepts. Then, the theoretical framework of the table will be expounded and several concrete examples of arguments based on these concepts will be analyzed in terms of the framework. Finally, the results of these analyses will be summarized and it will be indicated how they can be refined in further research related to the Periodic Table of Arguments.

Keywords: analogy, argument classification, argument from analogy, argument scheme, comparison, Periodic Table of Arguments, similarity.
\end{abstract}

\section{Introduction}

Viewed historically, the status of arguments based on the concept of analogy and similarity (and adjacent concepts such as comparison, equality, metaphor, parallel, proportion, and resemblance) is a much debated issue. ${ }^{1}$ Some scholars have defended the point of view that such arguments cannot be conceived in terms of deductive reasoning processes and are therefore fallacious per definition or should not even count as arguments at all. Other scholars have maintained that, exactly because the argument from analogy is based on a different reasoning process, it should be given an independent status and should be evaluated by using other criteria than those for determining deductive validity.

In present-day argumentation theory, scholars usually subscribe to the latter opinion in that they agree that supporting a standpoint by mentioning an analogous or similar case cannot be reduced to deduction or induction. But this agreement on the independent status of arguments from analogy 
does not imply that there is any consensus on the basic constituents of such arguments, the specific characteristics of the various sub types, or their relation to other types of argument. On the contrary, scholars have provided widely differing descriptions of this type of argument, thereby reflecting the state-of-the-art in argument classification. For concerning the central notion 'argument', there is a co-existence of a great variety of idiosyncratic taxonomies, many of which lack an explicit or clear rationale for distinguishing between the different types of argument.

The Periodic Table of Arguments is a new method for describing and classifying arguments that integrates the traditional dialectical accounts of argument schemes and fallacies and rhetorical accounts of the means of persuasion (logos, ethos, pathos) into a comprehensive framework. The table is based on an explicit and clear rationale for distinguishing between different types of argument. But it is not completely clear, as yet, how arguments based on the concept of analogy, similarity and adjacent concepts fit into its theoretical framework. The aim of this paper, then, is to analyze the systematic place of such arguments within the table and to describe the main characteristics of the sub types that can be distinguished.

The paper is structured as follows. First, I shall give a short overview of existing descriptions and classifications of arguments based on analogy, similarity, and adjacent concepts (Section 2). Next, I shall expound the theoretical framework of the Periodic Table of Arguments (Section 3) and analyze several concrete examples of arguments based on the concepts just mentioned in terms of that theoretical framework (Section 4). Finally, I shall summarize the results of these analyses and indicate some possibilities for further research and application of the table (Section 5).

\section{Existing accounts of arguments based on analogy}

Analogy as a type of reasoning and argument has been studied extensively in a great many academic fields. Apart from logic and argumentation theory, these fields include AI, ethics, law, psychology, and the natural sciences (Guarini et al., 2009). In the following discussion of existing accounts of arguments based on analogy, similarity, and adjacent concepts, I shall concentrate on those that belong to the field of argumentation theory.

According to Bartha, the author of the entry on analogy and analogical reasoning in the Stanford Encyclopedia of Philosophy, an analogical argument is 'an explicit representation of a form of analogical reasoning that cites accepted similarities between two systems to support the conclusion 
that some further similarity exists' (2016: 1). On the basis of this description and an inventory of examples taken from geometry, the history of science, and legal reasoning, he then states that such an argument is inductive in nature and has the following basic form:

$\begin{array}{ll}\text { premise } & S \text { is similar to } T \text { in certain (known) respects } \\ \text { premise } & S \text { has some further feature } Q \\ \text { conclusion } & T \text { also has the feature } Q\end{array}$

Figure 1. The general form of an analogical argument (Bartha 2016: 8)

While Bartha analyses this form of the argument from analogy as an argument based on induction, Juthe, in an introduction to his overview of proposals for classifying arguments by analogy, states that 'it has always been controversial whether arguments by analogy are a genuine class of arguments irreducible to the standard pattern of inductive or deductive arguments' (2016b: 62-63). The picture of the state-of-the-art that emerges from the overview itself is that scholars in argumentation theory have provided a large number of accounts of arguments by analogy, sometimes listed under alternative names such as the argument based on comparison or the argument from similarity, and sometimes accompanied by a list of sub types such as the argument from example, the argument by parallel, or the argument from normative analogy.

Apart from indicating the differences of opinion regarding the independence of the argument from analogy and its reducibility to arguments based on induction or deduction, Juthe also points out that argumentation theorists do not agree as to the perspective and the criteria from which to make further distinctions between types and sub types of the argument from analogy. As is clear from his extensive survey of the literature, depending on the choice of perspective and criteria, scholars make distinctions between comparing factual cases and hypothetical cases, a priori analogy and inductive analogy, predictive and proportional analogy, same-domain analogies and different-domain analogies, and between analogy of proportionality and analogy of attribution (2016a: 23-68).

Another much debated issue pertains to the acceptability, reasonableness or validity of arguments from analogy. Regarding this issue, some scholars take the position that all arguments based on analogy are fallacious (Garssen \& Kienpointner, 2011: 39). Others acknowledge the argumentative value of literal analogies, but have their reservations when it comes to figurative ones. According to Garssen, the latter scholars 'dismiss the figurative 
analogy as a form that has no probative force and only serves illustrative purposes' (2009: 134). At the other end of the scale, Wisdom (1991) has defended the opinion that all arguments, including those that reach their conclusion by means of deductive and inductive reasoning, are ultimately based on analogical reasoning (Juthe, 2016b: 63-65). All in all, analogical reasoning, as well as the forms of argument that are based on it, is deemed anything in between being inherently flawed and being the foundation of all kinds of reasoning.

Some of the differences between the accounts of arguments based on analogy can be traced back to the interpretation of the term 'analogy'. According to Walton, for instance, it is a widely accepted view that 'in order for something to qualify as an argument from analogy it must have one premise asserting that there is a similarity between two cases' (2014: 23). Other scholars, such as Perelman and Olbrechts-Tyteca, would rather call such an argument an 'argument from comparison' and interpret 'analogy' as a resemblance of structures that can be represented in the form $\mathrm{A}: \mathrm{B}=\mathrm{C}: \mathrm{D}$ and that is related to the notion of 'metaphor' (1969: 372, 399). The situation gets even more complicated as soon as concepts such as 'comparison', 'equality', 'parallel', 'proportion', and 'resemblance' are brought to the table.

In the introduction to a recent collection of articles on the argument by analogy, Ribeiro writes that regarding its analysis and representation there are 'deep divides and disagreements between theorists and contemporary researchers' (2014: 1). According to him, 'this does not mean [...] that the study of argument by analogy has not made significant and undeniable progress in the past decades. [...] The variety and plurality of evaluations of the argument by analogy must [...] be envisaged as a sign of wealth' (ibidem).

In my view, the situation regarding the description and classification of arguments based on analogy, similarity, and adjacent concepts is exemplary of the general state-of-the-art in argument classification and is less unproblematic than Ribeiro wants us to believe. Apart from differences between the dialectical and rhetorical approaches to argument, which conceptualize argument in very different ways, there are also differences within each of the two approaches. Although, for example, the 'new dialectic' (Walton et al., 2008) and 'pragma-dialectics' (van Eemeren \& Grootendorst, 2004), as the names of these approaches indicate, share a lot of common starting points, their accounts of argument schemes and the associated critical questions are theoretically disparate. Thirdly, there are problems inherent in specific accounts. The pragma-dialectical approach just mentioned, for instance, suffers from 
several inconsistencies, one of them being that the argument from example is identified as a sub type of symptomatic argumentation while it instantiates the argument scheme of argumentation based on a comparison (Hitchcock \& Wagemans, 2011: 194).

The existence of these theoretical issues in argument classifications is not without practical consequences. Since scholars work with idiosyncratic conceptualizations of the types of argument, the analyses they make are not directly comparable to one another. Further, in cutting-edge research such as argument mining and other computational applications, there is a need for a classification of argument that uses formal(izable) language and strong distinctive criteria.

The development of the Periodic Table of Arguments can be seen as an attempt to overcome the problems just mentioned by creating a systematic and comprehensive framework for argument classification. The table provides a theoretically sound and practically useful alternative to the existing multitude of incomplete, informal and sometimes even inconsistent taxonomies of argumentative techniques. ${ }^{2}$

\section{The theoretical framework of the Periodic Table of Arguments}

The Periodic Table of Arguments is a factorial typology of argument that is based on a theoretical framework consisting of the following three partial characterizations of argument.

(I) Predicate and subject arguments. This characterization draws on the differences and similarities between the statements that function as the conclusion and the premise of the argument. In short, the statements of predicate arguments contain the same subject $(a)$ and different predicates ( $X$ and $Y$ ), while their argumentative mechanism is based on a relationship between these predicates, and the statements of subject arguments contain different subjects ( $a$ and $b$ ) and the same predicate $(X)$, while their argumentative mechanism is based on a relationship between these subjects.

The general form of predicate arguments is ' $a$ is $X$, because $a$ is $Y$ '. An example is 'He was driving fast, because he left a long trace of rubber on the road'. In this case, the statements contain the same subject, 'He', and the argumentative mechanism is based on the relationship between the different predicates, the relationship between 'leaving a long trace of rubber on the road' and 'driving fast'. 
The general form of subject arguments is ' $a$ is $X$, because $b$ is $X$ '. An example is 'Biking on the lawn is forbidden, because walking on the lawn is forbidden'. In this case, the statements contain the same predicate, 'being forbidden', and the argumentative mechanism is based on a relationship between the different subjects, the relationship between 'walking on the lawn' and 'biking on the lawn'.

(II) First-order and second-order arguments. The two examples just mentioned can both be characterized as first-order arguments, because their argumentative mechanisms are based on a direct relationship between either the predicates or the subjects of the statements that function as the conclusion and the premise of the argument. Second-order arguments are different because their argumentative mechanism is based on an indirect relationship between these elements.

The general form of second-order predicate arguments is ' $a$ is $X$, because $a$ is $X$ is $Z$ '. An example is 'We only use $10 \%$ of our brain, because Einstein said so'. In this case, if we add 'is true' as a predicate to the conclusion and replace ' $a$ is $X$ ' by ' $q$ ', we can reformulate the general form as ' $q$ is true, because $q$ is $Z$ ' and it becomes clear that the argumentative mechanism is based on the relationship between the predicates 'being said by Einstein', and 'being true'.

The general form of second-order subject arguments is ' $a$ is $X$, because $b$ is $Y$ '. An example is 'Many truths turn out to be incredible to mortals, because false statements are persuasive among mortals' ${ }^{3}$ In this case, if we add 'is true' to both statements and replace ' $a$ is $X$ ' by $q$ and ' $b$ is $Y$ ' by ' $r$ ', we can reformulate the general form as ' $q$ is true, because $r$ is true', and it becomes clear that the argumentative mechanism is based on the relationship between the subjects $q$ and $r$, which can be specified as a twofold opposition between the terms of these propositions.

(III) Specific combinations of types of statements. Using the distinction between statements of policy $(\mathrm{P})$, statements of value $(\mathrm{V})$, and statements of fact (F) as a starting point, arguments can be characterized as instantiating one of the nine different combinations of types of statements (PP, PV, PF, VP, VV, VF, FP, FV, FF). The argument 'The government should invest in jobs, because this will have a positive impact on the economy', for instance, links a statement of policy $(\mathrm{P})$ expressed in the conclusion to a statement of fact $(\mathrm{F})$ expressed in the premise and can therefore be characterized as a 'PF argument'.

When taken together, these three partial characterizations of argument constitute a theoretical framework that allows for $2 \times 2 \times 9=36$ different types of arguments (see Figure 2). 


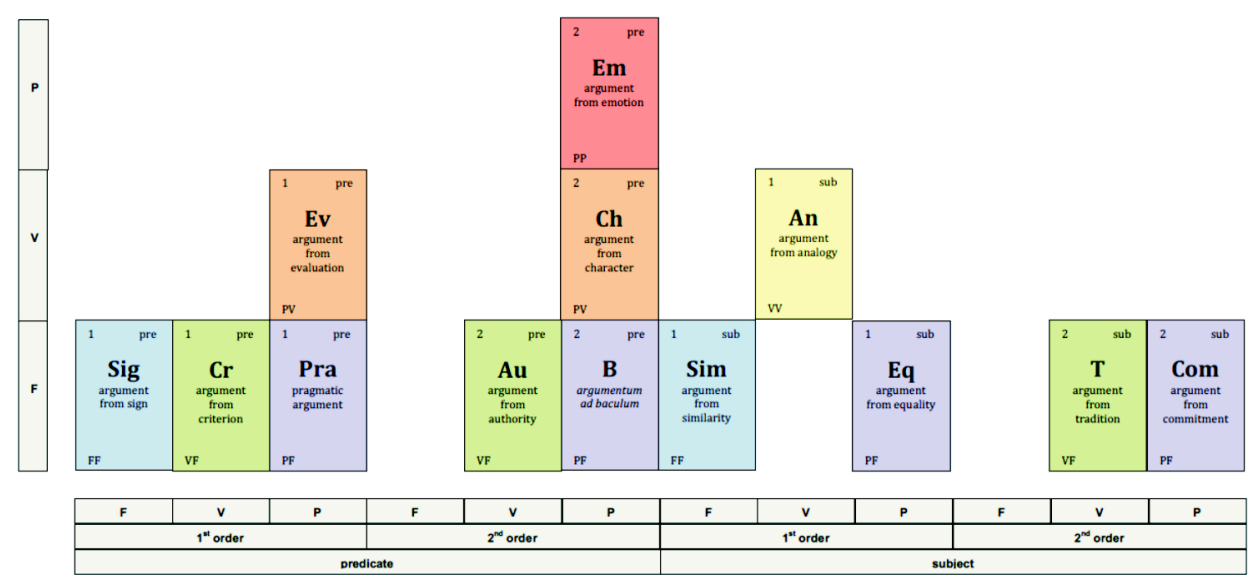

Figure 2. The Periodic Table of Arguments as depicted in Wagemans (2016: 10)

Each type of argument distinguished within this table has its own systematic name that can be used for the purpose of identifying real life examples of arguments. 'He was driving fast, because he left a long trace of rubber on the road', for instance, can be identified as a first-order predicate argument linking two statements of fact (1 pre FF).

In addition to this systematic name, some types of argument can also be given a trivial name on the basis of similarities with descriptions found in the existing dialectical and rhetorical taxonomies of argument. The example just mentioned, for instance, is traditionally known as the 'argument from sign'. In the version of the Periodic Table of Arguments depicted above, it is therefore symbolically represented as 'Sig' (see the lower left corner of the table in Figure 2). In other cases, the systematic characteristics of a specific type of argument are known, but a corresponding traditional description has not (yet) been found. An example is the first-order predicate argument linking a statement of fact with a statement of policy (1 pre FP), which is therefore represented by an open spot (see the upper left corner of the table in Figure 2).

\section{Differentiating arguments based on analogy}

In order to further differentiate arguments based on analogy, similarity and adjacent concepts within the theoretical framework of the table, I turn now to analyzing a number of concrete examples. For each of them, I identify the type of argument and propose a trivial name. 
A first example deals with the possibility of there being life on Mars. Positive standpoints about this issue are often defended by pointing at similarities with the planet we currently inhabit. The conclusion and the premise of such an argument can be expressed as follows:

\section{Example 1}

There is life on Mars, because there is life on Earth.

After reconstruction of the statements, the argument can be seen as an instantiation of the form ' $a$ is $X$, because $b$ is $X$ ', where ' $a$ ' stands for 'Mars', ' $b$ ' for 'Earth', and ' $X$ ' for 'being populated by living beings'. Since both the conclusion and the premise are statements of fact, the argument can be identified as a first-order subject argument linking a fact to another fact ( 1 sub FF). According to the theory behind the table, the argumentative mechanism of such arguments is based on the relation between the two subjects. This relation can be described as a relation of factual similarity. An indication for this being the case is to be found in the way in which the argumentative mechanism is further supported. Conspiracies.net (2017), for instance, mentions the following:

There are many similarities between Mars and Earth:

- The length of a day on Mars is similar to a day on Earth

- The tilting of its axis is similar to Earth's, meaning it experiences seasons in the same way Earth does.

- Mars has Polar Ice caps.

Since the support of the acceptability of the relation between the subjects consists of listing a number of factual similarities between Mars and Earth, I propose this argument to be given the trivial name 'argument from similarity'.

Another interesting example of an argument from analogy is given by Garssen: 'The evolutionary biologist Richard Dawkins argues that punishment is, scientifically speaking, out of date. He points out that it makes no sense to punish a car when it refuses to start and that it is equally irrational to punish criminals, because in their case something is broken as well: they come from poor families, received poor education or have poor genes.' (2009: 133). The conclusion and the premise of this argument can be reconstructed as follows:

\section{Example 2}

Punishing criminals is irrational, because it makes no sense to punish a car when it refuses to start. 
Like the previous example, this argument can be seen as an instantiation of the form ' $a$ is $X$, because $b$ is $X$ ', where ' $a$ ' stands for 'punishing criminals', ' $b$ ' for 'punishing a broken car', and ' $X$ ' for 'being irrational'. The difference lies in the type of statements expressed in the conclusion and the premise, which are not statements of fact but of value. Within the theoretical framework of the table, the argument can therefore be identified as a first-order subject argument linking a value to another value (1 sub VV). The argumentative mechanism behind such arguments is based on the relation between the two subjects, which can be described as a relation of analogy. As the co-text of the argument indicates, the arguer intends to convey that criminals can be 'broken' like cars can be broken. The support of the acceptability of this analogous application of the term to criminals consists of mentioning a number of shortcomings: 'they come from poor families, received poor education or have poor genes'. I therefore propose this argument to be given the trivial name 'argument from analogy'.

According to a poll carried out by Dutch television programme EenVandaag, 62 percent of Dutch people think that King Willem-Alexander of the Netherlands, his wife Queen Máxima, and his mother Princess Beatrix should pay taxes just like every other citizen (Politiek.tpo.nl, 2017). The conclusion and the premise of this argument can be expressed as follows:

\section{Example 3}

Dutch royals should pay taxes, since every other Dutch citizen pays taxes.

Like the previous examples, this is a first-order subject argument, but this time, yet another combination of types of statements is instantiated: the conclusion consists of a statement of policy and the premise of a statement of fact ( 1 sub PF). Since the argumentative mechanism behind such arguments is that the subject of the conclusion should be treated equally as the subject in the premise, I propose this argument to be given the trivial name 'argument from equality'.

A final example is found in the following fragment taken from a speech held by the former U.S. president Abraham Lincoln on June 9, 1864. Lincoln is defending the standpoint that he should not be replaced, given that the country is in the middle of a civil war. ${ }^{4}$ 


\section{Example 4}

I have not permitted myself, gentlemen, to conclude that I am the best man in the country; but I am reminded, in this connection, of a story of an old Dutch farmer who remarked to a companion once that 'it was not best to swap horses while crossing a stream'.

Taking into account the contextual information provided above, in this argument the conclusion 'One should not replace a president in the middle of a war' is defended by the premise 'One should not swap horses while crossing a stream'. The argument can be identified as a first-order subject argument linking a statement of policy to another statement of policy (1 sub PP). Since the argumentative mechanism of this argument is based on a comparison between the two situations, I propose 'argument from comparison' as the trivial name of this type of argument.

\section{Conclusion}

In this paper, I have indicated the systematic place of arguments based on analogy, similarity, and adjacent concepts within the Periodic Table of Arguments. The analysis of a number of concrete examples has shown that the arguments under scrutiny (1) can be viewed as first-order subject arguments and (2) can be further differentiated in a meaningful way by looking at the types of statements involved. As a result, we may conclude that in the theoretical framework of the table, at least the following main types of arguments can be distinguished that are based on an analogical reasoning process:

- 1 sub FF, which links a fact to another fact and can be given the trivial name 'argument from similarity' since its argumentative mechanism is based on the existence of one or more factual similarities between the two subjects of the statements involved;

- 1 sub VV, which links a value to another value and can be given the trivial name 'argument from analogy' since its argumentative mechanism is based on the subject of the conclusion being analogous to that of the premise;

- 1 sub PF, which links a policy to a fact and can be given the trivial name 'argument from equality' since its argumentative mechanism is based on the idea that the two subjects of the statements should be treated in the same way; 
- 1 sub PP, which links a policy to another policy and can be given the trivial name 'argument from comparison' because policies are usually more elaborate than simple entities and may involve multiple comparisons between characteristic elements of the two situations.

Given the factorial character of the classification, I do claim mutual exclusiveness for these four types. But, since their actual description belongs to the first results of explorative research into the existing dialectical and rhetorical descriptions and classifications of argument types, I do not expect them to be the only types of argument that are based on analogical reasoning processes. First of all, an extended study of the literature as well as experimental and computational research into the actual occurrence of types of arguments in persuasive discourse may show that some of the gaps in the present list of first-order subject arguments can be filled after all. And second, further research may give rise to a differentiation of each of the individual argument types distinguished within the theoretical framework of the table. When analyzing the specific distinctions made within the classical tradition of topics (topoi, loci), for instance, regarding the arguments a genere (from the genus), ex comparatione maiorum, minorum and parium (from the greater, the lesser, and the like degrees), it is possible to describe certain 'isotopes' of first-order subject arguments depending on the semantic or even ontological relations between the statements involved. ${ }^{5}$

As far as applications of this new classification of arguments are concerned, the Periodic Table of Arguments can, first of all, be used to carry out comparative research between existing classifications and to check individual classifications for internal consistency. When it comes to comparing different approaches to the types of arguments discussed in this paper, the overview given in Section 2 may function as a starting point. Second, since the table employs formal(izable), unified language to describe the types of argument, it can be used to systematically analyze, evaluate, and generate concrete argumentative discourse. Third and last, but definitively not least, the table may be used as theoretical input for developing computational applications such as tools for argument mining. For the latest results of these various types of research related to the table, please consult its official website at www.periodic-table-of-arguments.org.

\section{N O T E S}

1 This paper was presented as a keynote lecture at the PhiLang 2017 Conference held at the University of Łódź from May 12-14, 2017. 


\section{Jean H.M. Wagemans}

2 The summary of the problems related to existing classifications of argument and the reasons for developing a Periodic Table of Arguments in this section and the explanation of its theoretical framework in the next section of this paper are adapted versions of sections in Wagemans (2016b, 2018).

3 The example is mentioned by Aristotle in his description of the topos 'from opposites' in Rhetoric 1397a and is taken from Euripides' Thyestes, fragment 396 (see Kennedy 1991: 191).

4 This example and its analysis have been taken from Wagemans (2016a: 89).

${ }^{5}$ For the classical tradition of topics, see e.g. Rubinelli (2009) and van Eemeren et al. (2014: 86-94).

\section{R E F E R E N C E S}

Bartha, P. (2016). Analogy and analogical reasoning. In E.N. Zalta (Ed.), The Stanford Encyclopedia of Philosophy (Winter 2016 Edition), URL = https:// plato.stanford.edu/archives/win2016/entries/reasoning-analogy/.

Conspiracies.net (2017). Mars conspiracies: Is there life on Mars? URL $=\mathrm{www}$. conspiracies.net/mars-conspiracy-life/.

Eemeren, F.H. van, Garssen, B.J., Krabbe, E.C.W., Snoeck Henkemans, A.F., Verheij, H.B., \& Wagemans, J.H.M. (2014). Handbook of Argumentation Theory. Dordrecht: Springer.

Eemeren, F.H. van, \& Grootendorst, R. (2004). A systematic theory of argumentation: The pragma-dialectical approach. Cambridge: Cambridge University Press.

Garssen, B.J. (2009). Comparing the incomparable: Figurative analogies in a dialectical testing procedure. In F.H. van Eemeren \& B.J. Garssen (Eds.), Pondering on problems of argumentation: Twenty essays on theoretical issues (pp. 133-140). Dordrecht: Springer.

Garssen, B.J., \& Kienpointner, M. (2001). Figurative analogy in political argumentation. In E.T. Feteris, B.J. Garssen \& A.F. Snoeck Henkemans (Eds.), Keeping in touch with pragma-dialectics (pp. 39-58). Amsterdam: Benjamins.

Guarini, M., Butchart, A., Smith, P.S., \& Moldovan, A. (2009). Resources for research on analogy: A multi-disciplinary guide. Informal Logic, 29(2), 84197.

Hitchcock, D.L., \& Wagemans, J.H.M. (2011). The pragma-dialectical account of argument schemes. In E.T. Feteris, B.J. Garssen \& A.F. Snoeck Henkemans (Eds.), Keeping in touch with pragma-dialectics (pp. 185-205). Amsterdam: Benjamins.

Juthe, L.J.A. (2016a). Argumentation by analogy: A systematic analytical study of an argument scheme. Dissertation University of Amsterdam.

Juthe, L.J.A. (2016b). Classifications of arguments by analogy, Part I: A comprehensive review of proposals for classifying arguments by analogy. Cogency, 8(2), 51-99. 
Kennedy, G.A. (1991). Aristotle, On rhetoric: A theory of civic discourse. New York / Oxford: Oxford University Press.

Perelman, C., \& Olbrechts-Tyteca, L. (1969). The new rhetoric: A treatise on argumentation. Translated by J. Wilkinson \& P. Weaver. Notre Dame: University of Notre Dame Press.

Politiek.tpo.nl (2017). Ook dit jaar vindt de Nederlander dat de Oranjes belasting moeten betalen. URL = http://politiek.tpo.nl/2017/04/25/ook-dit-jaarvindt-de-nederlander-dat-de-oranjes-belasting-moeten-betalen/.

Ribeiro, H.J. (2014). Introduction. In idem (Ed.), Systematic approaches to argument by analogy. Cham / Heidelberg / New York / Dordrecht / London: Springer.

Rubinelli, S. (2009). Ars topica: The classical technique of constructing arguments from Aristotle to Cicero. Dordrecht / Boston: Springer.

Wagemans, J.H.M. (2016a). Analyzing metaphor in argumentative discourse. Rivista ltaliana di Filosofia del Linguaggio, 10(2), 79-94.

Wagemans, J.H.M. (2016b). Constructing a Periodic Table of Arguments. In P. Bondy \& L. Benacquista (Eds.), Argumentation, Objectivity, and Bias: Proceedings of the 11th International Conference of the Ontario Society for the Study of Argumentation (OSSA), 18-21 May 2016 (pp. 1-12). Windsor, ON: OSSA.

Wagemans, J.H.M. (2018). Assertoric syllogistic and the Periodic Table of Arguments. In S. Oswald \& D. Maillat (Eds.), Argumentation and Inference: Proceedings of the 2nd European Conference on Argumentation, Fribourg 2017 (Volume I, pp. 573-588). London: College Publications.

Walton, D. (2014). Argumentation schemes for argument from analogy. In H.J. Ribeiro (Ed.), Systematic approaches to argument by analogy. (pp. 23-40). Cham / Heidelberg / New York / Dordrecht / London: Springer.

Walton, D., Reed, C., \& Macagno, F. (2008). Argumentation schemes. New York: Cambridge University Press.

Wisdom, J.O. (1991). Proof and explanation: The Virginia lectures. Edited by Stephen F. Barker. Lanham: University Press of America. 